REVISÕES

\title{
Patologia de Sementes Florestais: Danos, Detecção e Controle, uma revisão
}

\author{
João José Dias Parisi ${ }^{1} \oplus$, Alvaro Figueredo dos $\operatorname{Santos}^{2}{ }^{\circ}$, Claudio José Barbedo ${ }^{3}$, Priscila Fratin Medina ${ }^{4}$
}

${ }^{1}$ Instituto Florestal / Estação Experimental de Tupi, Rodovia Luiz de Queiroz, Km 149, CEP 13428-000, Piracicaba-SP, Brasil. ${ }^{2}$ Embrapa Florestas, Estrada da Ribeira, Km 111, 83411-000 Colombo, PR, Brasil. ${ }^{3}$ Instituto de Botânica, Núcleo de Pesquisa em Sementes, Av. Miguel Stéfano 3687, 04301-902 São Paulo-SP, Brasil. ${ }^{4}$ Instituto Agronômico de Campinas, Centro de Grãos e Fibras, Laboratório de Sementes, Av. Barão de Itapura, 1481, 13020-902 Campinas-SP, Brasil.

Autor para correspondência: João José Dias Parisi (joao parisi2000@yahoo.com.br)

Data de chegada: 30/11/2017. Aceito para publicação em: 26/06/2018.

$10.1590 / 0100-5405 / 188545$

\section{RESUMO}

Parisi, J.J.D., Santos, A.F.; Barbedo, C.J.; Medina, P.F.. Patologia de Sementes Florestais: Danos, Detecção e Controle, uma revisão. Summa Phytopathologica, v.45, n.2, p.129-133, 2019.

A demanda por sementes com boa qualidade fisiológica e sanitária, com o propósito de produzir mudas sadias de espécies florestais nativas, cresceu nos últimos anos, em função da restauração de florestas, recuperação de áreas degradadas, recomposição de paisagens e matas ciliares. Poucas pesquisas têm sido desenvolvidas na área de sanidade de sementes, direcionadas à identificação de fungos, à participação destes no processo de deterioração, à condução de testes de patogenicidade e à transmissão de patógenos. Também há poucos estudos sobre tratamento e conservação de sementes das espécies de interesse e, consequentemente, de preservação da diversidade vegetal, especialmente das sementes nativas de matas tropicais. A grande diversidade de espécies dessas formações vegetais demanda esforços ainda maiores para que se possa obter sucesso na identificação e controle dos principais patógenos associados às sementes de espécies florestais.

Palavras-chave: Patógeno; tratamento de sementes; sementes florestais, armazenamento.

\section{ABSTRACT}

Parisi, J.J.D., Santos, A.F.; Barbedo, C.J.; Medina, P.F.. Pathology of Forest Tree Seeds: Damage, Detection and Control, a Review. Summa Phytopathologica, v.45, n.2, p.129-133, 2019.

The demand for seeds of high physiological and sanitary quality, for the production of healthy seedlings of native forest tree species, has grown in recent years due to forest restoration, recovery of degraded areas and restoration of riparian forests and landscapes. Scarce research has been performed in the field of seed health with the aim of identifying fungal species, detecting their participation in the deterioration process, and conducting pathogenicity tests and pathogen transmission. There are also few studies on seed treatment and conservation for the species of interest and, consequently, on plant diversity preservation, especially for seeds of trees native to tropical forests. The high species diversity of these plant formations demands even greater efforts to succeed in identifying and controlling the major pathogens associated with seeds of forest species.

Keywords: Pathogen, seed treatment, forest seeds, storage.

Diante da importância histórica, ecológica, econômica e cultural de se conservar e explorar, de maneira sustentável, os recursos naturais, investimentos originaram-se a partir de políticas públicas de conservação, criação e manejo de áreas protegidas (57), resultando no desenvolvimento e na implantação de diversas tecnologias com o intuito de recuperação dos remanescentes florestais (48) e na formação de bancos de germoplasma (11). Segundo esses, no Brasil, a maioria dos reflorestamentos com espécies nativas é realizada com mudas produzidas por sementes, o que permite manter ou ampliar a base genética das futuras populações regeneradas.

$\mathrm{Na}$ fase de maturação dos frutos, as deficiências minerais e hídricas do solo e a incidência de pragas e doenças podem impedir as sementes de atingirem a qualidade máxima disponível no potencial genético e, por conseguinte, acelerar a sua deterioração no armazenamento. Tal fato é amplamente conhecido para sementes de espécies agrícolas, mas pouco estudado em sementes de espécies florestais $(42,43)$.

A curta longevidade restringe o período de disponibilidade de sementes de diversas dessas espécies, exigindo que a semeadura seja efetuada logo após a obtenção. Essa limitação pode concentrar a oferta de mudas em determinadas épocas do ano ou, ainda, tornálas disponíveis em épocas inadequadas de plantio, o que dificulta a inclusão de espécies arbóreas tropicais nos programas de recuperação de áreas nativas degradadas (3). Dentre os fatores que contribuem para a curta longevidade das sementes de espécies florestais, a falta de conhecimento das condições sanitárias e fisiológicas mais favoráveis para a conservação da viabilidade figura entre os principais.

A maioria dos trabalhos com enfoque em patologia de sementes florestais foi realizada na Índia, no Canadá, nos Estados Unidos e na África, abordando principalmente as coníferas (51). No Brasil, as pesquisas com esse enfoque foram iniciadas por Lasca et al. (21), que 
detectaram fungos dos gêneros Alternaria, Ascochyta, Botryodiplodia, Diplodia, Fusarium, Helminthosporium e Pestalotiopsis em sementes importadas de Pinus spp. Na década de 1980, Mucci \& Lasca (32) e Carneiro (7) constataram, em sementes de espécies florestais nativas, diversos fungos potencialmente patogênicos às plantas cultivadas. A partir dessa época, diversos trabalhos científicos têm sido desenvolvidos, mas poucas publicações que sistematizem e discutam os resultados dessas pesquisas (51). Esta revisão tem o objetivo de agrupar e discutir os resultados disponíveis sobre danos, detecção e controle dos fitopatógenos associados com sementes florestais.

\section{Danos em sementes e mudas}

Em florestas tropicais há um número muito maior de espécies de insetos e microrganismos do que de espécies vegetais, que vivem em equilíbrio dinâmico com as plantas, assumindo o papel de pragas e doenças, quando esses ecossistemas são desequilibrados (16). Umidade e temperatura elevadas favorecem o crescimento e desenvolvimento de patógenos, tornando as sementes das espécies nativas dessas regiões vulneráveis ao ataque dos mesmos (33).

A disseminação e a manutenção da viabilidade dos fungos são favorecidas pela associação destes às sementes, em comparação à associação com outros propágulos vegetais (25), pois as sementes frequentemente apresentam elevados teores de proteínas, carboidratos e minerais, o que facilita a colonização dos tecidos pelos patógenos, estendendo-se até os primeiros estádios do desenvolvimento das plântulas (28). Segundo esses, o inóculo pode atingir flores, frutos e sementes, originar-se em outras plantas, em outro local de infecção da planta-mãe ou no solo e disseminar-se por meio do vento, a água, os insetos ou a própria semente, durante a colheita e o beneficiamento.

A inclusão de padrões sanitários em sementes de florestais, visando ao controle da qualidade de sementes e mudas produzidas, pode ser uma medida estratégica para dificultar a introdução em novas áreas e disseminação de patógenos (58).

A morte das sementes pode ser causada pela ação de enzimas e toxinas produzidas pelos patógenos, antes do início da germinação, durante este processo ou após a emergência das plântulas. Também pode ocorrer debilitação das plantas, caracterizada por podridões radiculares, bloqueio no transporte de água e nutrientes e colonização sistêmica, resultando em desenvolvimento reduzido, porte inadequado e sintomas de deficiência nutricional. Pouco vigorosas, as plantas tornam-se suscetíveis a qualquer tipo de estresse: seca, elevada umidade relativa do ar, temperatura alta ou baixa e ataque de pragas e doenças (27).

Nas espécies florestais, uma das doenças mais conhecidas é o tombamento de mudas, causado por Cilindrocladium sp., Fusarium spp. e Rhizoctonia solani, que também podem destruir as sementes em germinação (pré-emergência), ou as plântulas recém-emergidas, atacando os tecidos tenros e suculentos (pós-emergência). Os sintomas da doença manifestam-se no colo da muda, com aspecto encharcado no início, adquirindo posteriormente coloração escura, resultante da degeneração dos tecidos, culminando no tombamento e morte da muda. Tais fungos têm sido comumente encontrados em associação com sementes florestais $(37,38,42,43,44,53$ e 58).

O número de pesquisas sobre patogenicidade, transmissão e danos causados por patógenos às plântulas de espécies florestais ainda é pequeno, destacando-se Colletotrichum sp. e Pestalotiopsis sp. em sementes de aroeira pimenteira (39); Alternaria alternata, Fusarium spp., Aspergillus spp., Phoma sp. e Phomopsis sp. em sementes de ipê amarelo e roxo (5); Fusarium sp. e Pestalotiopsis sp. em sementes de cedro (4); Cladosporium cladosporioides e Pestalotiopsis sp. em pau-
Brasil (24); Alternaria sp., Colletotrichum sp., Fusarium sp. Rhizoctonia sp. em sementes de paineira e cedro (22, 23); Penicillium sp. e Fusarium sp. em sementes de espécies de Eugenia e Inga vera (36, 44, 45); Cladosporium sp., Pestalotia sp. e Macrophomina sp. em sementes de murta (47), Phomopsis sp. em angico vermelho (60). Constataram-se ainda indícios da transmissão de fungos dos frutos para as sementes de tauva (39), além da correlação positiva entre índice de sementes mortas e porcentagem de fungos incidentes (38). Estudos realizados por Dhingra et al. (12) e Parisi et al. (39) comprovaram a infecção latente de fungos inoculados em sementes florestais. Mais relatos e metodologia específica para testes de patogenicidade podem ser consultados no livro de patologia de sementes florestais (52) e na revisão Patologia de sementes de espécies florestais no Brasil (50).

No armazenamento, fungos podem depreciar a qualidade das sementes, por causarem a colonização do embrião, descoloração e apodrecimento, com reflexos tanto na viabilidade como no valor comercial e nutritivo; aumento da taxa de ácidos graxos e rancificação de óleos; aquecimento da massa de sementes, gerando aumento da taxa respiratória e a aceleração da deterioração (25), fato comprovado em sementes de Inga vera (42). Dentre os fungos de armazenamento, destacam-se os dos gêneros Aspergillus e Penicillium, adaptados a ambientes com umidade relativa do ar alta (65 a 90\%). Aspergillus flavus é, geralmente, a espécie mais destrutiva (14). Mittal (30), trabalhando com sementes de Cedrus deodara, observou que Rhizopus oryzae, Penicillium canadense e A. flavus reduziram a porcentagem de germinação e e o vigor das plântulas.

Além disso, a maioria das espécies de florestas tropicais é originária de regiões úmidas e suas sementes são liberadas com elevado teor de água. A alta sensibilidade à perda de água faz necessário o armazenamento com alto grau de umidade, favorecendo o ataque de microrganismos e a germinação indesejada das sementes. O uso de baixas temperaturas poderia inibir esses problemas, mas muitas sementes recalcitrantes sofrem danos por temperaturas próximas ou abaixo de zero (59).

Para sementes recalcitrantes, geralmente armazenadas úmidas, em temperaturas superiores a $0^{\circ} \mathrm{C}(20)$, a ocorrência de fungos de armazenamento constitui-se em um dos principais fatores prejudiciais à conservação da viabilidade (31). Poucos trabalhos conseguiram comprovar os danos ocasionados pelos fungos, devido, principalmente à baixa longevidade das sementes dessas espécies. Há dúvidas se a deterioração das sementes oferece condições para os fungos proliferarem ou se estes causam a debilitação das sementes. Berjak (1) considerou que ambos os fatores estão simultaneamente envolvidos. Oliveira et al. (36) e Parisi et al. (44 e 45) constataram a influencia dos fungos na conservação de sementes de espécies de Eugenia e Inga vera respectivamente.

De acordo com Parisi et al. (44 ou 45), o desenvolvimento de Pestalotiopsis sp., Phoma sp., Phomopsis diachenii e Colletotrichum gloeosporioides em sementes de $I$. vera ocorre simultaneamente à deterioração dos embriões, em função do alto grau de umidade, com que são armazenados, diferindo do padrão clássico definido com base em sementes ortodoxas, que se caracteriza pela perda de viabilidade ao longo do tempo, de fungos como esses, considerados como de campo, devido ao baixo grau de umidade dessas sementes. Segundo Barbedo et al. (2) a pesquisa deve ser direcionada à ampliação do período de maturação de sementes recalcitrantes, mantendo-as ligadas à plantamãe até que o processo de maturação tenha sido concluído, ou até um estádio, o mais próximo possível do ponto de maturidade fisiológica. Dessa forma os danos causados pelos microrganismos poderiam ser mais facilmente comprovados. 


\section{Detecção}

De acordo com Machado (25), os patógenos podem ser transportados nas sementes na forma de misturas, aderidos à superfície ou internamente, nos cotilédones e no embrião. Os métodos que têm sido usados para detecção de fungos em sementes florestais são a incubação através do método do papel de filtro (blotter test) e o plaqueamento em meio de batata-dextrose-ágar (BDA), ambos conduzidos em regime de 12 horas de luz alternada (40). Dentre estes, o método mais usado é o do papel de filtro com um período de incubação das sementes, que varia de 7 a 10 dias, e temperatura entre 18 e $26^{\circ} \mathrm{C}$ (53). Santos et al. (54) constataram maior incidência do fungo Lasiodiplodia theobromae através do método de papel de filtro em relação ao plaqueamento em BDA. Nascimento et al. (33) verificaram que o método do papel de filtro com congelamento em relação ao sem congelamento permitiu a identificação de Fusarium moniliforme e Alternaria alternata em sementes de amendoim-bravo (Pterogynenitens). Costa Jr. et al. (8) e Silva et al. (56) têm relatado métodos seletivos para detecção de Fusarium spp. em sementes de pupunha e pínus, respectivamente.

Outros fatores de variação nos resultados de avaliação de fungos em sementes estão relacionados com o tempo, o produto e a sua concentração (etanol, hipoclorito de sódio, hidróxido de sódio, entre outros) utilizados para a assepsia superficial das sementes. Em geral, o hipoclorito de sódio é o mais utilizado.

Em testes de germinação, onde a desinfestação superficial não é utilizada, fungos como Rhizopus sp. podem contaminar todas as amostras contidas no germinador, dificultando assim a interpretação das avaliações.

Os testes baseados no desenvolvimento de sintomas em plântulas (40) e nas mudas florestais, utilizando substrato esterilizado, além de fornecer resultados sobre a emergência das plântulas podem também trazer conhecimentos sobre a transmissão dos fungos via sementes $(8,56)$.

\section{Controle}

O tratamento pode ser utilizado para eliminar ou reduzir os fungos das sementes florestais, com o objetivo de obtenção de mudas com boa qualidade sanitária e silvicultural. As sementes tratadas estarão protegidas contra patógenos associados às próprias sementes ou presentes no solo, evitando também a disseminação de microorganismos patogênicos para áreas ainda não contaminadas (41). Os resultados de sanidade de sementes florestais obtidos nas pesquisas realizadas com tratamento de sementes, envolvendo métodos químicos, físicos e/ou biológicos, podem ser destacados pelo seu impacto, atual ou futuro, no contexto da manutenção da qualidade das sementes, através da identificação e do controle de fungos.

Apesar de não existirem produtos registrados, a eficiência do tratamento químico de sementes das espécies florestais com os produtos captan, thiram, thiabendazole, tiofanato metílico, carbendazin + thiram, carboxin + thiram e captan + tiofanato metílico, tiofanato metílico + clorotalonil têm sido relatada experimentalmente por vários autores $(6,8,13,17,22,24,36,44$ e 45). Os tratamentos físicos, como a termoterapia em água e o condicionamento osmótico têm sido alvo de pesquisas recentes também em sementes florestais $(18,19,35$, $36,46)$. Esses podem conferir grande eficiência quanto à erradicação do patógeno e à germinação das sementes de Eugenia spp. (36), mas dependem da sensibilidade diferencial ao estresse hídrico entre a semente e o patógeno, proporcionado pelo binômio tempo-temperatura e dos potenciais hídricos da solução. De acordo com Hopkins \& Mcquilken (15), o crescimento de Pestalotiopsis sydowiana em baixos potenciais hídricos pode ajudar a explicar a alta incidência e a severidades de doenças fúngicas, observadas em plantas que se desenvolvem em condições de estresse hídrico.

Quanto ao tratamento biológico, na área agrícola já existem produtos comerciais produzidos a partir de isolados de fungos, como por exemplo, à base de espécies de Trichoderma, podendo ser utilizados no controle dos patógenos encontrados nas sementes e no solo. No entanto, faltam tais informações para as sementes florestais (41).

A exploração da atividade biológica de compostos secundários produzidos pela própria planta também pode originar uma alternativa para o controle de patógenos associados às sementes, com a vantagem de redução, tanto dos gastos, quanto do impacto ambiental, causado pelos agroquímicos (9).

Extratos etanólicos das raízes e extratos aquosos das folhas frescas de Ottonia martiana apresentaram atividade antifúngica, em relação ao desenvolvimento micelial, dos patógenos Fusarium oxysporum, Colletotrichum acutatum e Rhizoctonia sp., principalmente nas concentrações mais elevadas (10). Segundo Schwan-Estrada et al. (55), o extrato bruto ou óleo essencial de plantas medicinais da flora nativa têm apresentado efeito positivo no controle de fitopatógenos, tanto por sua ação fungitóxica direta, inibindo o crescimento micelial e a germinação de esporos, quanto pela indução de fitoalexinas, indicando a presença de compostos com características de elicitores.

$\mathrm{O}$ efeito de diferentes extratos aquosos também foi analisado sobre sementes de açoita cavalo por Miethet al. (29), cujas maiores incidências fúngicas foram de Fusarium sp., Phoma sp., Cladosporium sp., Rhizoctonia sp. e Colletotrichum sp. Segundo o autor, extratos de tabaco controlaram a incidência de Fusarium sp. E Cladosporium sp., enquanto os extratos aquosos de pitanga e cinamomo inibiram Rhizoctonia sp. e Colletotrichum sp. Além destes, extratos aquosos das serrapilheiras de Pinus elliott e Eucalyptus grandis inibiram o desenvolvimento de fungos, sem causarem danos à germinação de sementes de Copaifera sp (34).

Outra linha abordada pela comunidade de pesquisadores e ambientalistas é a dos teores de água críticos para secagem das sementes florestais, gerando contribuição fundamental ao prolongamento da viabilidade no armazenamento. Contudo, essa medida isolada não garante a conservação, sendo necessária também à redução da temperatura e a identificação dos fungos associados a essas sementes e de seus possíveis danos. Destacam-se os resultados obtidos em pau brasil (Caesalpinia echinata), que possibilitam a conservação da capacidade germinativa das sementes durante pelo menos dois anos, pela redução do teor de água a $8-10 \%$, acondicionamento em embalagem permeável e armazenamento a $-18^{\circ} \mathrm{C}$. (26). Estas informações são essenciais para o estabelecimento de estratégias de conservação ex situ do pau brasil, contribuindo para evitar a redução do germoplasma da espécie. Fungos de importância agrícola foram identificados nessas sementes e, por se tratarem de patógenos em potencial, merecem ser alvo de maiores estudos de patogenicidade.

\section{CONSIDERAÇÕES FINAIS}

A utilização de sementes e mudas de espécies florestais nativas cresceu nos últimos anos em função da demanda para restauração de florestas, recuperação de áreas degradadas, recomposição de paisagens e matas ciliares, de maneira que o uso de sementes de espécies florestais nativas com qualidade fisiológica e sanitária para a produção de mudas sadias é de extrema importância. Assim, novas pesquisas 
abordando a patogenicidade, os fatores que atuam na transmissão e os danos causados por patógenos às plântulas de espécies florestais, bem como as transformações bioquímicas e celulares que ocorrem durante o armazenamento de sementes florestais são essenciais para o conhecimento e controle das doenças e para a conservação de sementes e, conseqüentemente, da preservação da diversidade vegetal. Pelo aqui exposto, verifica-se que poucas pesquisas estão sendo desenvolvidas nessa área. Contudo, a grande diversidade de espécies demanda maiores de informações para possibilitar o sucesso na identificação e controle dos patógenos associados às sementes de espécies florestais.

\section{REFERÊNCIAS}

1. Berjak, P. The role of microorganisms in deterioration during storage of recalcitrant and intermediate seeds. In: Ouédraogo, A.S.; Poulsen, K.; Stubsgaard, F. Intermediate/recalcitrant tropical forest tree seeds: proceedings of a working on improved methods for handling and storage of intermediate/ recalcitrant tropical forest tree seeds. Rome: IPGRI, 1995. v.1, p.121-126.

2. Barbedo, C.J.; Centeno, D.C.; Ribeiro, R.C.L.F. Do recalcitrant seeds really exist? Hoehnea, São Paulo, v.40, n.4, p.583-593, 2013.

3. Barbedo, C.J.; Marcos Filho, J. Tolerância à dessecação em sementes. Acta Botanica Brasilica, Belo Horizonte, v.12, n.2, p.145-164, 1998.

4. Benetti, S.C.; Santos, A.F.; Medeiros, A.C.; Jaccoud Filho, D.S. Levantat mento de fungos em sementes de cedro e avaliação da patogenicidade de Fusarium sp. e Pestalotia sp. Pesquisa Florestal Brasileira, Colombo, v.38, p.81-5 2009.

5. Botelho, L.S.; Moraes, M.H.D.; Menten, J.O.M. Fungos associados às sementes de ipê-amarelo (Tabebuia serratifolia) e ipê-roxo (Tabebuia impetiginosa): incidência, efeito na germinação e transmissão para as plântulas. Summa Phytopathologica, Botucatu, v.34, n.4, p.343-348, 2008.

6. Bulow, J.F.W.; Carmona, R.; Parente, T.V. Armazenamento e tratamento de sementes de pitanga-vermelha-do-cerrado (Eugenia calycina). Pesquisa Agropecuária Brasileira, Brasília, v.29, n.6, p.961-970, 1994.

7. Carneiro, J.S. Micoflora associada à sementes de essências florestais. Fitopatologia Brasileira, Brasília, v.11, p.557-566, 1986.

8. Costa Jr., J.C.; Santos, A.F.; Francisco, L.; Silva, C.N.; Tessmann, D.J. Qualidade sanitária e fisiológica, métodos de detecção de Fusarium spp. e tratamento de sementes de pupunheira. Ciência Florestal, Santa Maria, v.26, n.4, p.1119-1131, 2016.

9. Coutinho, W.M.; Araújo, E.; Magalhães, F.H.L. Efeitos de extratos de plantas anacardiáceas e dos fungicidas químicos benomyl e captan sobre a micoflora e qualidade fisiológica de sementes de feijoeiro (Phaseolusvulgaris L.). Ciência e Agrotecnologia, Lavras, v.23, n.3, p.560-568, 1999.

10. Cunico, M.M.; Carvalho, J.L.S.; Andrade, C.A.; Miguel, O.G.; Miguel, M.D.; Auer, C.G.; Grigoletti Júnior, A.; Côcco, L.C.; Yamamoto, C. I. Atividade antifúngica de extratos brutos de Ottonia martiana Miq., Piperaceae. Visão Acadêmica, Curitiba, v.7, n.2, p. 13, 2006.

11. Davide, A.C.; Silva, E.A.A. Sementes florestais. In: Davide, A.C.; Silva, E.A.A. (Ed.). Produção de sementes e mudas de espécies florestais. Lavras: UFLA, 2008. p.11-81.

12. Dhingra, O.D.; Maia, C.B.; Lustosa, D.C.; Mesquita, J.B. Seed born e pathogenic fungi affect seedling quality of red angico (Anadenantheramacrocarpa) trees in Brazil. Phytopathology, Saint Paul, v.150, p.451-455, 2002.

13. Faiad, M.G.R.; Salomão, A.N.; Padilha, L.S. Levantamento de populações fúngicas associada às sementes de Spondias tuberosa Anacardiaceae e sua redução através de tratamento fungicidas. Summa Phytopathologica, Botucatu, v.21, n.3/4, p.245-248, 1995.

14. Hocking, A.D.; Banks, J.H. Effects of phosphine fumigation on survival and growth of storage fungi in wheat. Journal of Stored Products Research, Pergamom, v.27, n.2, p.115-120, 1991.

15. Hopkins, K.E.; Mcquilken, M.P. Characteristics of Pestalotiopsis associated with hardy ornamental plants in the UK. European Journal of Plant Pathology, Netherlands, v.106, p.77-85, 2000.

16. Kageyama, P. A biodiversidade como ferramenta em agroecossistemas. In: Barbosa, L.M.; Santos Junior, N.A. (Org.). A botânica no Brasil: pesquisa, ensino e políticas públicas ambientais. São Paulo: Sociedade Botânica do Brasil, 2007. p.83-87.
17. Ferreira, F.A. Patologia florestal: principais doenças florestais no Brasil. Viçosa: Sociedade de Investigações Florestais, 1989. 570p.

18. Françoso, C.F.; Barbedo, C.J. Tratamentos osmóticos e térmicos para controle de fungos em sementes de grumixameira (Eugenia brasiliensisLam.) e pitangueira (Eugenia uniflora L.). Hoehnea, São Paulo, v.41, p.541-552, 2004.

19. Françoso, C.F.; Barbedo, C.J. Osmotic and heat treatment son control of fungi associated with seeds of Eugenia brasiliensis and E. pyriformis (Myrtaceae). Journal of Seed Sciences, Londrina, v.38, n.3, p.195-203, 2016.

20. Goldbach, H. Imbibed storage of Melicoccus bijugatus and Eugenia brasiliensis using abscisic acid as a germination inhibitor. Seed Science and Technology, Zurich, v.7, n.3, p.403-406, 1979.

21. Lasca, C.C.; Sampaio, A.S.; Cintra, A.F. Condições fitossanitárias de sementes importadas de Pinus spp. O Biológico, São Paulo, v.27, p.287292, 1971.

22. Lazarotto, M.; Muniz, M.F.B.; Santos, A.F. Detecção, transmissão, patoł genicidade e controle químico de fungos em sementes de paineira (Ceiba speciosa). Summa Phytopathologica, Botucatu, v.36, p.105-112, 2010.

23. Lazarotto M.; Muniz, M.F.B.; Beltrame, R.; Santos, A.F.; Maciel, C.G.M.; Longhi, S.J.L. Sanidade, transmissão via semente e patogenicidade de fungos em sementes de Cedrela fissilis procedentes da região sul do Brasil. Ciência Florestal, Santa Maria, v.22, n.3, p.493-503, 2012.

24. Lisbôa-Padulla, T.; Moraes, M.H.D.; Barbedo, C.J.; Borges, I.F.; Menten, J.O.M.; Pascholati, S.F. Detecção de fungos em sementes de pau-brasil (Caesalpinia echinata) coletadas durante sua formação e dispersão. Revista Brasileira de Sementes, Londrina, v.32, p.154-159, 2010.

25. Machado, J.C. Tratamento de sementes: fundamentos e aplicações. Brasília: MEC-ESAL-FAEPE, 1988. 106p.

26. Mello, J.L.O.; Parisi, J.J.D.; Figueiredo-Ribeiro, R.C.L.; Barbedo, C.J. Meia década de armazenamento de sementes de Pau-Brasil. In: Congresso Nacional de Botânica, 60, 2009, Feira de Santana. Resumo, Brasília: Sociedade Botânica do Brasil, 2009. 1 CD-ROM.

27. Menten, J.O.M. Patógenos em sementes: detecção, danos e controle químico. Piracicaba: ESALQ/FEALQ, 1991. 321p.

28. Menten, J.O.M.; Bueno, J.T. Transmissão de patógenos pelas sementes. In: Soave, J.; Wetzel, M.V.S. Patologia de sementes. Campinas: Fundação Cargill, 1987. 480 p.

29. Mieth, A.T.; Piveta, G.; Pacheco, C.; Hamann, F.A.; Rodrigues, J.; Muniz, M.F.B.; Blume, E. Influência de extrato vegetal na qualidade sanitária e fisiológica em sementes de Lueheadivaricata (Açoita-cavalo). Revista Brasileira de Agroecologia, Porto Alegre, v.2, n.2, p.1240-1242, 2007.

30. Mittal, R.K. Studies on for micoflora and its control on the seeds of some forest trees: Cedrus deodara. The Malaysian Forester, Kepong Selangor, v.61, n.1, p.197-201, 1983.

31. Mycock, D.J.; Berjak, P. Fung a contaminants associated with several homoiohydrous (recalcitrant) seedspecies. Phytophylactica, Saskatoon, v.22, p.413-418.

32. Mucci, E.S.F.; Lasca, C.C. Flora fúngica de sementes de essências florestais nativas. Fitopatologia Brasileira, Brasilia, v.11, n.2, p.352353, 1986.

33. Nascimento, W.M.O.; Cruz, E.D.; Moraes, M.H.D.; Menten, J.O.M. QualQ idade sanitária e germinação de sementes de Pterogynenitens Tull. (Leguminosae - Caesalpinioideae). Revista Brasileira de Sementes, Pelotas, v.28, n.1, p.149-153, 2006

34. Oliveira, C.F.; Júnior, N.A.S.; Parisi, J.J.D. Efeito alelopático de extratos aquosos das serapilheiras de duas espécies arbóreas exóticas sobre a germinação e a incidência de fungos em sementes de Copaifera Langsdorffil Desp. (Leguminosae - Caesalpinioideae). In: Congresso Nacional de Botânica, 58., 2007, São Paulo. Resumo, 2007. Brasília: Sociedade Botânica do Brasil, 2007. 1 CD-ROM.

35. Oliveira, M.D.; Nascimento, L.C.; Alves, E.U.; Golçalves, E.P.; Guedes, R.S.; Silva Neto, J.J. Qualidade sanitária e fisiológica de sementes de Amburana cearensis A.C. Smith submetidas à termoterapia e tratamento químico. Acta Scientiarum Agronomy, Maringá, v.33, n.1, p.45-50, 2011.

36. Oliveira, C.F.; Oliveira, D.C.; Parisi, J.J.D.; Barbedo, C.J. Deterioração de sementes de espécies brasileiras de Eugenia em função da incidência e do controle de fungos. Revista Brasileira de Sementes, Londrina, v.33, n.3, p.520-532, 2011

37. Parisi, J.J.D.; Martins, M.C.; Sales, W.R.M. Qualidade sanitária e fisiológica de sementes de espécies florestais do estado de São Paulo, Campinas, SP. In: Simpósio Brasileiro de Patologia de Sementes, 8., 2004, Resumos. João Pessoa, 2004. p.203. 
38. Parisi, J.J.D; Andrade, F.A.; Coutinho, E.L.; Botosso, M.C.; Martins, M.C. Sales, W.R.M. Qualidade sanitária de sementes e mudas de espécies arbóreas nativas do Estado de São Paulo. Informativo Abrates, Londrina, v.15, n.1/3, p.3, 2005.

39. Parisi, J.J.D.; Santos, A.F. dos. Descrição de doenças, detecção e controle de patógenos transmitidos por sementes de espécies florestais. In: Simpósio Brasileiro de Patologia de Sementes, 9., 2006, Passo Fundo. Anais. Cidade de publicação, Editora, 2006. p..

40. Parisi, J.J.D.; Santos, A.F. Métodos convencionais de detecção de fungos em sementes. In: Santos, A.F.; Parisi, J.J.D.; Menten, J.O. Patologia de sementes florestais. Colombo: Embrapa Florestas, 2011. cap.4, p.49-61.

41. Parisi, J.J.D.; Santos, A.F.; Menten, J.O. Tratamento de sementes florestais. In: Santos, A.F.; Parisi, J.J.D.; Menten, J.O. Patologia de sementes florestais. Colombo: Embrapa Florestas, 2011. cap.8, p. 105-114.

42. Parisi, J.J.D. Situação e contribuições da patologia de sementes de espécies florestais de interesse no Brasil. In: Congresso Brasileiro de Fitopatologia, 45., 2012, Manaus. Anais. Brasília: Tropical Plant Pathology, 2012. 1 CD ROM.

43. Parisi, J.J.D. Transmissão de patógenos através de sementes e mudas de espécies florestais nativas. In: Congresso Brasileiro de Sementes, 18. 2013, Florianópolis. Palestras e Resumos. Londrina: Informativo Abrates, 2013. 1 CD-ROM.

44. Parisi, J.J.D.; Biagi, D.J.; Barbedo, C.J.; Medina, P.F. Viability of Inga vera Willd. subsp. affinis (DC.) TD Penn. embryos according to the maturation stage, fungal incidence, chemical treatment and storage. Journal of Seed Science, Londrina, v.35, n.1, p.70-76, 2013.

45. Parisi, J.J.D.; Biagi, D.J.; Barbedo, C.J.; Medina, P.F. Fungicide and drying effects on the viability of recalcitrant seeds of Inga vera subsp. affinis. Tropical Plant Pathology, Viçosa, v.41, p.177-182, 2016.

46. Piveta, G.; Lazzaroto, M.; Mezzomo, R.; Santos, R.F.; Webwe, M.N.; Muniz, M.B. Efeito do tratamento térmico na qualidade sanitária e fisiológica de sementes de Lafoensia pacari St. Hil. Revista Brasileira e Agroecologia, v.4, n.2, p.1653-1657, 2009.

47. Rego, S.S.; Santos, A.F.; Nogueira, A.C.; Kuniyoshi, Y.S. Detection, transmission and pathogenicity of fungi on Blepharocaly $\mathrm{x}$ salicifolius (H.B.K.) Berg. seeds. Revista Brasileira de Sementes, Londrina, v.34, n.1, p.9-13, 2012.

48. Reis, A.; Bechara, F.C.; Espindola, M.B.; Vieira, N.K.; Souza, L.L. Restaus ração de áreas degradadas: a nucleação como base para incrementar os processos sucessionais. Natureza \& Conservação, Curitiba, v.1, n.1, p. 28-36, 2003.
49. Salomão, A.N.; Padilha, L.S. Avaliação preliminar da germinabilidade e da micoflora associada às sementes de Geniba americana em diferentes estágios de maturação. Brasília: Embrapa Recursos Genéticos e Biotecnologia, 2006. (Circular técnica, 50).

50. Santos, A.F.; Eckstein, B.; Muniz, M.F.B.; Carmo, A.L.M.;Auer, C.G.; Jaccoud Filho, D.S.; Parisi, J.J.D. Patologia de sementes de espécies florestais no Brasil. Revisão Anual de Patologia de Plantas, Passo Fundo, v.23, p.193-211, 2015.

51. Santos, A.F.; Grigoletti Júnior, A.; Auer, C.G. Transmissão de fungos por sementes de espécies florestais. Floresta, Colombo, v.30, n.1/2, p.119$128,2000$.

52. Santos, A.F.; Parisi, J.J.D.; Menten, J.O.M. Patologia de sementes florestais. 1.ed. Colombo: Embrapa Florestas, 2011. 236p.

53. Santos, A.F.; Parisi, J.J.D. 2004. Estado da arte e perspectivas da patologia de sementes florestais no Brasil. In: Simpósio Brasileiro de Patologia de Sementes, 8., 2004, João Pessoa. Anais.

54. Santos, M.F.; Ribeiro, R.C.W.; Faiad, M.G.R.; Salomão, S.M. Fungos associados às sementes de baru (Dipteryxalata Vog.). Revista Brasileira de Sementes, Brasília, v.19, n.1, p.135-139, 1997.

55. Schwan-Estrada, K.R.F.; Stangarlin, J.R.; Cruz, M.E.S. Uso de extratos vegetais no controle de fungos fitopatogênicos. Floresta, Curitiba, v.1, n.2, p.129-137, 2000.

56. Silva, T.W.R.; Santos, A.F.; Auer, C.G.; Tessmann, D.J. Métodos de detecção, transmissão e patogenicidade de Fusarium spp. em sementes de Pinus taeda. Ciência Florestal, Santa Maria, v.27, n.1, p.73-84, 2017.

57. Tabarelli, M.; Pinto, L.P.; Silva, J.M.C.; Hirota, M.M.; Bedê, L.C. Desafios e oportunidades para a conservação da biodiversidade na Mata Atlântica brasileira. Megadiversidade, Belo Horizonte, v.1, n.1, p.132-138, 2005.

58. Vechiato, M.H.; Parisi, J.J.D. Importância da qualidade sanitária de sementes florestais na produção de mudas. Divulgação Técnica. Biológico, São Paulo, v.75, n.1, p.27-32, 2013.

59. Vieira, A.H.; Martins, E.P.; Pequeno, P.L.L.; Locatelli, M., Souza, M.G. Técnicas de produção de sementes florestais. Comunicado técnico Embrapa, Rondônia, p.1-4, 2001.

60. Walker, C.; Maciel, C.G.; Bovolini, M.; Pitorini, P.; Schultz, C.; Marlove, M.F.B. Transmissão e patogenicidade de Phomopsis sp. associadas às sementes de angico-vermelho (Parapiptadenia rígida Benth.). Floresta e Ambiente, Seropédica, v.20, n.2, p.216-222, 2013. 